\title{
Assessment of biological characteristics of mesenchymal stem cells labeled with superparamagnetic iron oxide particles in vitro
}

\author{
JUN-HUI SUN ${ }^{1}$, YUE-LIN ZHANG ${ }^{1}$, SU-PING QIAN ${ }^{2}$, XIAO-BO YU ${ }^{1}$, \\ HAI-YANG XIE ${ }^{1}$, LIN ZHOU ${ }^{1}$ and SHU-SEN ZHENG ${ }^{1}$
}

\begin{abstract}
${ }^{1}$ Key Laboratory of Combined Multi-Organ Transplantation, Ministry of Public Health, Key Laboratory of Organ Transplantation, Zhejiang Province, Division of Hepatobiliary and Pancreatic Surgery, Department of Surgery, First Affiliated Hospital, School of Medicine, Zhejiang University, Hangzhou 310003; ${ }^{2}$ Molecular Imaging Platform, Zhejiang-California International Nanosystems Institute, Zhejiang University, Hangzhou 310003, P.R. China
\end{abstract}

Received July 27, 2011; Accepted October 13, 2011

DOI: $10.3892 / \mathrm{mmr} .2011 .637$

\begin{abstract}
Mesenchymal stem cell (MSC) transplantation provides a novel strategy for the treatment of human disease. MR imaging (MRI) is able to track transplanted stem cells labeled with superparamagnetic iron oxide (SPIO) in vivo. However, the effect of SPIO upon labeled MSCs remains unclear on a cellular level. In this study, the biological characteristics of rat MSCs labeled with home-synthesized SPIO particles were evaluated. The MSCs were isolated from the bone marrow of 5 adult Sprague-Dawley rats and labeled with home-synthesized SPIO particles at a final iron concentration of $20 \mu \mathrm{g} / \mathrm{ml}$. Labeled MSCs were confirmed with Prussian blue staining and transmission electron microscopy. The quantity of iron per cell was determined by atomic absorption spectrometry. Cell viability, proliferation, membranous antigen and multiple differentiation ability were compared between labeled and unlabeled MSCs. The rat MSCs were effectively labeled and the labeling efficiency was approximately $100 \%$, as revealed by Prussian blue staining. The SPIO particles located in the endosomal vesicles of the MSCs were confirmed by transmission electron microscopy. No significant differences were observed in cell viability, proliferation, membranous antigen and multiple differentiation ability between the labeled and unlabeled MSCs $(\mathrm{P}>0.05)$. In conclusion, MSCs are able to be effectively labeled by home-synthesized SPIO particles without influencing their main biological characteristics.
\end{abstract}

Correspondence to: Professor Shu-Sen Zheng, Key Laboratory of Combined Multi-Organ Transplantation, Ministry of Public Health, Key Laboratory of Organ Transplantation, Zhejiang Province, Division of Hepatobiliary and Pancreatic Surgery, Department of Surgery, First Affiliated Hospital, School of Medicine, Zhejiang University, 79 Qingchun Road, Hangzhou, Zhejiang 310003, P.R. China

E-mail: shusenzheng@zju.edu.cn

Key words: mesenchymal stem cell, superparamagnetic iron oxide particles, cell labeling

\section{Introduction}

As a potential interventional procedure, stem cell transplantation provides a new strategy for the treatment of incurable human diseases and organ failure (1-3). The plasticity of bone marrow mesenchymal stem cells (MSCs) has been intensively investigated, since this approach generates less ethical and social controversies. The use of bone marrow MSCs also offers several advantages, including ease of collection and rapid in vivo and in vitro repopulation. Numerous studies have shown that MSCs are capable of forming functional components of organ tissues, including the kidney, heart and liver (1-4).

A non-invasive in vivo technique that permits evaluation of the efficiency of transplantation and the potential migration of transplanted cells would prove to be an essential tool for treatment assessment. To better understand the mechanisms of the cell therapy, in vivo monitoring of the cellular dynamics of the transplanted MSCs has been proposed. Recently, MR imaging (MRI) has proven to be effective in tracking the distribution of transplanted MSCs in vivo by way of labeling cells with superparamagnetic iron oxide (SPIO) particles (5-7).

However, it is not clear whether the SPIO labeling technique of MSCs is safe. Additionally, the effect of SPIO upon labeled cells remains unclear on a cellular level (8). Thus, the purpose of the present study was to investigate whether and how the labeling of MSCs with SPIO affects the biological characteristics of MSCs.

\section{Materials and methods}

Cell culture. This study was approved by our institutional Animal Use and Care Committee. MSCs were generated from the bone marrow of 5 adult Sprague-Dawley rats, aged 6 weeks and weighing 120-150 g. The bone marrow cells were obtained by flushing the rat femurs with phosphate-buffered saline (PBS), and then the monocyte layer was selected with density centrifugation and resuspended in low-glucose Dulbecco's modified Eagle's medium (DMEM; Gibco, Carlsbad, CA, USA) supplemented with $0.2 \mathrm{mmol} / \mathrm{ml} \mathrm{L}$-glutamine, 100 units 
of penicillin, $100 \mathrm{~g} / \mathrm{ml}$ streptomycin and $10 \%$ fetal bovine serum (SAFC, St. Louis, MO, USA). Prior to being planted in a $25 \mathrm{~cm}^{3}$ culture flask, the cells were grown in the culture solution, which was adjusted to $1 \times 10^{6} / \mathrm{ml}$ of the concentration, and then grown in the standard culture media at $37^{\circ} \mathrm{C}$ with $5 \% \mathrm{CO}_{2}$.

Cell labeling and identification of the labeled cells. The SPIO $\left(\mathrm{Fe}_{3} \mathrm{O}_{4}\right)$ nanoparticles were donated by Professor S.P. Qian (Molecular Imaging platform, Zhejiang-California International Nanosystems Institute, Zhejiang University, Hangzhou, China). The MSCs were grown in $25 \mathrm{~cm}^{3}$ flasks. The cells growing at Passage ${ }_{3}\left(\mathrm{P}_{3}\right)$ were transferred to the culture media containing SPIO nanoparticles for labeling. A concentration of $20 \mu \mathrm{g} / \mathrm{ml}$ iron was used for culture. The MSCs were incubated continuously for $12 \mathrm{~h}$ at $37^{\circ} \mathrm{C}$ in an incubator with $95 \%$ air per $5 \% \mathrm{CO}_{2}$.

The cells were harvested by removing the free SPIO nanoparticles washed with PBS four times. For the purpose of Prussian blue staining to identify the cell profile and intracellular iron particles, the cells were continuously incubated for 15 min with $2 \%$ potassium ferrocyanide in $6 \%$ hydrochloric acid, and then counterstained with nuclear fast red for $3 \mathrm{~min}$.

To measure the iron concentration within the cells, the cell suspension was dissolved in $37 \%$ hydrochloric acid and then assayed with a polarized atomic absorption spectrometer (Shengyang Huaguang HG-9602A, Shengyang, China). The measuring process was repeated three times, and the mean value was adopted. The distribution of the SPIO particles within the cells was revealed under electron microscopy. The harvested labeled MSCs were fixed at $4^{\circ} \mathrm{C}$ in $2.5 \%$ buffered glutaraldehyde for $1 \mathrm{~h}$, followed by $1 \%$ osmium tetroxide for $2 \mathrm{~h}$. The samples of these cells were examined with a transmission electron microscope (H600, Hitachi, Japan).

Cell viability, proliferation, membranous antigen and multiple differentiation capability. The cell viability, proliferation activity and differentiation capability of SPIO-labeled and unlabeled MSCs were evaluated and compared. All procedures were performed in triplicate.

Cell viability was assessed by trypan blue testing. Cell proliferation activity was observed under a light microscope (Axioscop, Zeiss, Germany). Furthermore, tetrazolium salt (MTT) assay was performed to assess the toxicity and the effect of SPIO labeling on MSC proliferation. The MSCs at $\mathrm{P}_{3}$ were grown in a 96-well plate at $10^{4}$ cells per well. SPIO solution at a final iron concentration of $20 \mu \mathrm{g} / \mathrm{ml}$ was added into 40 wells and the remaining 40 wells, to which SPIO was not added, served as a control. The absorbance values of unlabeled MSCs and SPIOlabeled MSCs were measured from day 1 to 5 of the culture process ( 8 wells per day). For the assay, $20 \mu \mathrm{l}$ MTT $(5 \mathrm{mg} / \mathrm{ml}$; Fluka, St. Gallen, Switzerland) was added into each well and incubated at $37^{\circ} \mathrm{C}$ in $5 \% \mathrm{CO}_{2}$ for $4 \mathrm{~h}$. A total of $150 \mu \mathrm{l}$ dimethyl sulfoxide (DMSO; Sigma-Aldrich, St. Louis, MO, USA) was added and the medium was stirred for $10 \mathrm{~min}$. When the indigo crystals (formazan crystals) were dissolved evenly in the medium, the light absorption value of each well was measured with a spectrophotometer (Model 680; Bio-Rad Laboratories, Inc., Hercules, CA, USA) using a 490-nm wavelength.

The expression of membranous antigen on labeled and unlabeled MSCs was detected by cytofluorimetric analysis with a flow cytometer (Becton Dickinson, San Jose, CA, USA). The primary mouse anti-rat monoclonal antibodies of anti-CD29, anti-CD45 and anti-CD90 (BD Pharmingen, San Diego, CA, USA) were employed. The multipotency of labeled and unlabeled MSCs was confirmed by their induction of osteogenic and adipogenic differentiations with specific differentiation media. Osteogenic differentiation was assessed using von Kossa staining, to show the mineralization, while adipogenic differentiation was visualized by means of oil red $\mathrm{O}$ staining of lipid vacuoles in the adipocytes differentiated from MSCs.

Statistical analyses. Statistical analyses were carried out using the SPSS $^{\circledR}$ statistical package, version 11.0 (SPSS Inc., Chicago, IL, USA) for Windows ${ }^{\circledast}$. Data are presented as the mean \pm standard deviation (SD). To compare the differences between the labeled and unlabeled MSCs in various tests, the Kruskal-Wallis rank sum test was used to determine the difference in absorbance of MTT. $\mathrm{P}<0.05$ was considered to be statistically significant.

\section{Results}

Morphological observation of the MSCs. In the present experiment, MSCs were obtained from rat bone marrow and purified by density centrifugation. When they were passaged to $\mathrm{P}_{3}$, the purified MSCs retained the pattern of uniform fibroblast-like cells (Fig. 1). The homogeneity of the cells reached approximately $99 \%$.

Cell labeling and identification of the labeled cells. Cells were stained with Prussian blue, and blue particles were observed within most labeled MSCs. The labeling rate was approximated to $100 \%$ (Fig. 2), while no blue particles were found in unlabeled cells. The iron quantification per cell measured by atomic absorption spectrometer was $17.5 \pm 2.2 \mathrm{pg}$. Transmission electron microscopy revealed the SPIO particles were located in the endosomal vesicles of labeled MSCs (Fig. 3).

Cell viability, proliferation, membranous antigen and multiple differentiation capability. Trypan blue exclusion testing revealed a mean viability of $98.1 \pm 1.1 \%$ for the SPIO-labeled MSCs. The mean viability of the unlabeled MSCs was $97.5 \pm 1.2 \%$. There were no significant differences between the labeled and unlabeled MSCs ( $\mathrm{P}>0.05)$. The aspects of labeled MSCs, including shape, figure and nucleolus, were no different from those of the unlabeled MSCs under the light microscope.

On days 1 to 5, the absorbence of the labeled MSCs was $0.185 \pm 0.006,0.201 \pm 0.007,0.251 \pm 0.018,0.299 \pm 0.011$ and $0.359 \pm 0.017$, respectively, while the absorbence of the unlabeled MSCs was $0.196 \pm 0.008,0.208 \pm 0.006,0.247 \pm 0.012$, $0.305 \pm 0.014$ and $0.362 \pm 0.018$, respectively. There was no significant difference in MTT absorbance values between the labeled and unlabeled MSCs at each time point $(\mathrm{P}>0.05)$.

The flow cytometric analysis revealed that the majority of

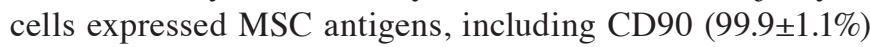
and CD29 (99.3 $\pm 1.5 \%)$, but did not express hematopoietic lineage marker CD45 $(1.7 \pm 0.1 \%)$. While the labeled MSCs also expressed CD90 $(99.5 \pm 1.6 \%)$ and CD29 $(94.4 \pm 2.1 \%)$, they did not express CD45 (3.0 $\pm 0.2 \%)$ (Fig. 4). There was no significant 


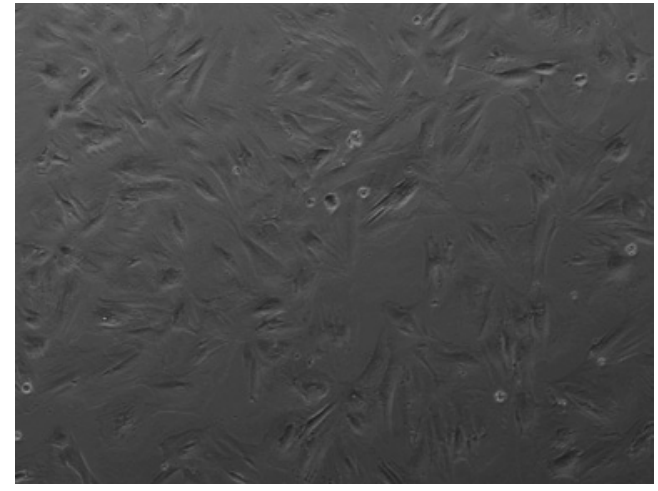

Figure 1. Under a light microscope (x200), the rat MSCs $\left(\mathrm{P}_{3}\right)$ appeared as fibroblast-like cells with slim bodies.

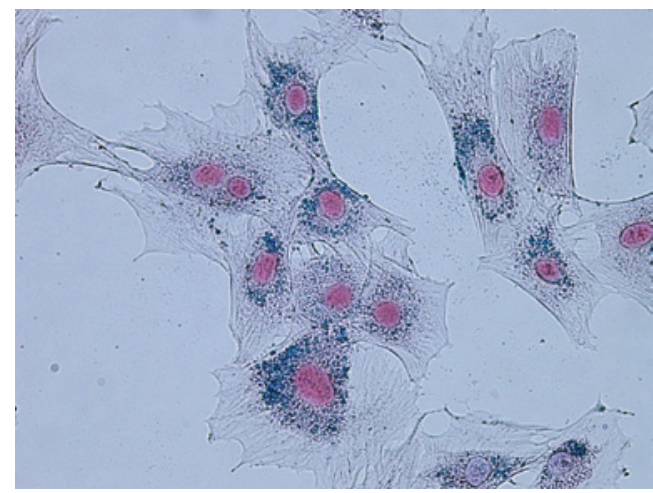

Figure 2. Prussian blue staining revealed intracytoplasmic blue particles that were clearly visible in most MSCs. The labeled rate reached approximately $100 \%$ (x200).

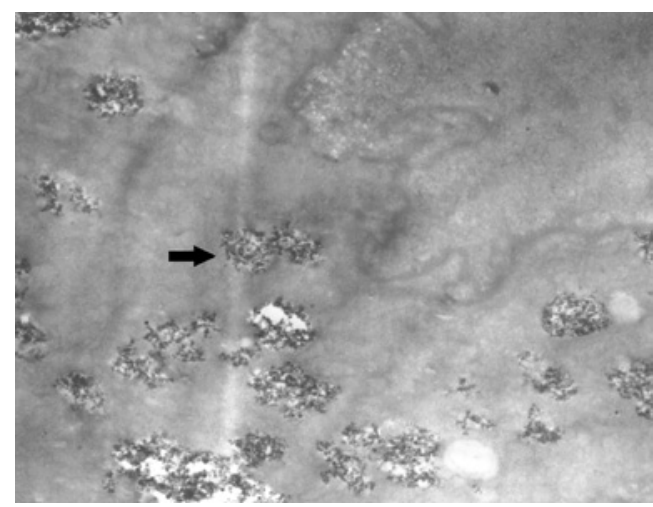

Figure 3. Transmission electron micrograph revealed that the endosoma vesicles containing the SPIO particles appear in black clusters (arrowhead) located in the MSCs outside the nucleus (original magnification, x10,000).

difference in antigen expression between the labeled and unlabeled MSCs ( $\mathrm{P}>0.05)$.

Labeled and unlabeled MSCs all showed positivity with von Kossa staining for mineralization nodules following osteogenic induction. Intracytoplastic lipid vesicles were observed by oil red $\mathrm{O}$ staining following adipogenic induction. The multipotency of the labeled MSCs was no different from that of the unlabeled MSCs.
A

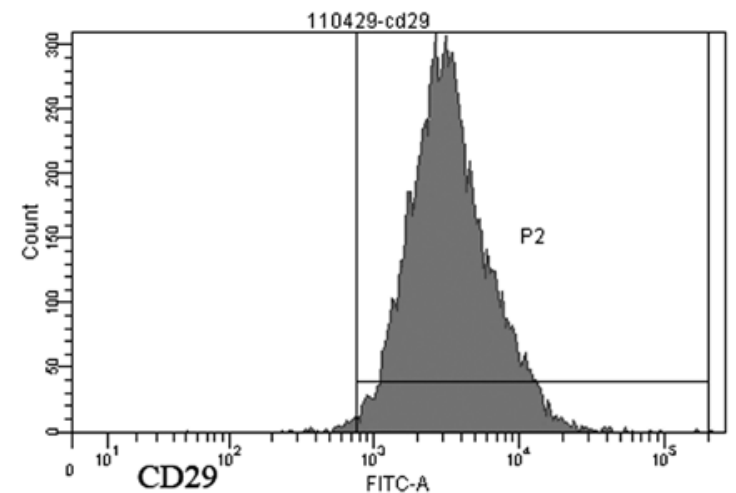

B

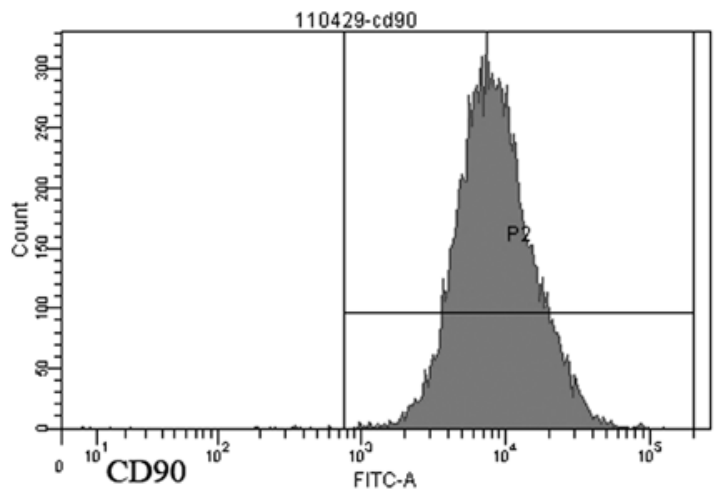

C

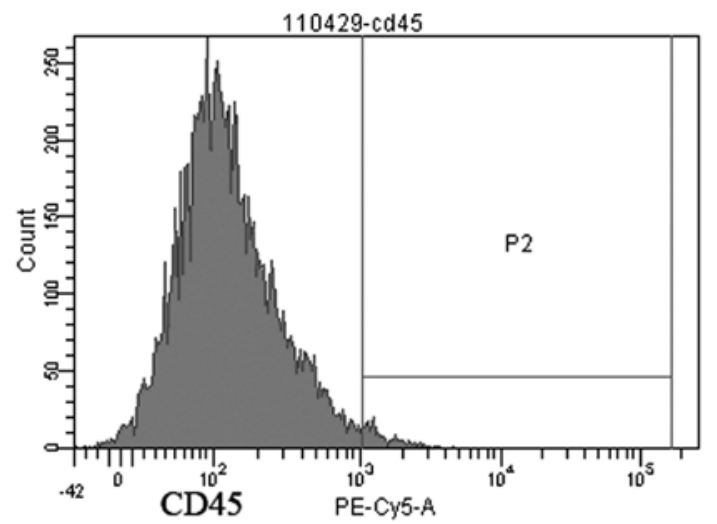

Figure 4. Flow cytometric analysis revealed that the SPIO-labeled MSCs, being homogeneous on $\mathrm{P}_{3}$, were uniformly positive for markers of MSCs such as surface proteins CD29 (A) and CD90 (B). In contrast, the MSCs were negative for markers of the hematopoietic lineage such as CD45 (C).

\section{Discussion}

Compared to organ transplantation, cell transplantation has the advantages of lower cost and risk $(9,10)$. Additionally, autologous cell transplantation avoids immunological rejection. Herein, there is no doubt that cell transplant treatment has a promising potential application in certain severe diseases, in particular the end-stage diseases, such as organ failure. Among the various types of transplanted cells, bone marrow MSCs have been previously demonstrated to hold better prospects in terms of their practical applicability for transplantation, since both in vitro and in vivo studies have revealed their capability of transdifferentiation (11-13).

In recent years, to monitor the transplanted MSCs, several non-invasive in vivo molecular imaging tracking techniques have been developed, including nuclear medicine, optical 
imaging and MRI (14-16). The MR technique holds promising advantages due to its wide imaging window, high temporal and spatial resolution, and good contrast, without ionic radiation. With the cell labeling technique using specific agents, the presence of labeled cells is easily visualized by MRI. Among the MR tracing agents, SPIO appears to gain its popularity (17) since SPIO particles in nanometeric size have strong penetrating capability, which makes it possible to cause a signal change in MR imaging at a super-low tracer concentration (nmol level). Some investigators have already demonstrated the feasibility of in vivo tracking of transplanted MSCs by labeling them with magnetic SPIO particles (18-20). Obviously, the low efficiency of loading the SPIO particles into cells and the cytotoxicity of previously formulated particles limit their usage as an image probe (21). It has been demonstrated by several groups that SPIO particles have little toxicity and few side effects on cell proliferation and activity $(21,22)$. However, certain study results have indicated the inhibitory effect of SPIO particles on MSC differentiation and its signaling mechanism (23). To conclude, the effect of SPIO upon labeled MSCs on a cellular level remains uncertain. Therefore, it is essential to demonstrate efficient labeling without deleterious effects on MSC cell phenotype and differentiation capacity.

It has been demonstrated in this study that rat MSCs are able to be effectively labeled by these home-synthesized SPIO particles. The results of the present study also indicated that there is no difference in cell viability, proliferation, membranous antigen and multiple differentiation capability between SPIO-labeled MSCs and unlabeled MSCs. Therefore, the labeling SPIO particle has little influence on the biological properties of MSCs. Moreover, these results may be significant in the use of MRI to track and precisely localize the transplanted, magnetically labeled MSCs by in vivo methods.

\section{Acknowledgements}

This project was supported by the National Natural Science Foundation of China (no. 30901446), the Program for Innovative Research Team of Science and Technology of Zhejiang Province (no. 2009R50038), the Medical Health Fund of Zhejiang Province (no. 2008A053) and the Programme of Chinese Medical Science of Zhejiang Province (no. 2009CB040).

\section{References}

1. Dai W, Hale SL, Martin BJ, Kuang JQ, Dow JS, Wold LE, Kloner RA: Allogeneic mesenchymal stem cell transplantation in postinfarcted rat myocardium: short- and long-term effects. Circulation 112: 214-223, 2005.

2. Orlic D, Kajstura J, Chimenti S, et al: Mobilized bone marrow cells repair the infarcted heart, improving function and survival. Proc Natl Acad Sci USA 98: 10344-10349, 2001.

3. Kassem M, Kristiansen M and Abdallah BM: Mesenchymal stem cells: cell biology and potential use in therapy. Basic Clin Pharmacol Toxicol 95: 209-214, 2004.
4. Sato Y, Araki H, Kato J, et al: Human mesenchymal stem cells xenografted directly to rat liver are differentiated into human hepatocytes without fusion. Blood 106: 756-763, 2005.

5. Qian H, Yang H, Xu W, et al: Bone marrow mesenchymal stem cells ameliorate rat acute renal failure by differentiation into renal tubular epithelial-like cells. Int J Mol Med 22: 325-332, 2008.

6. Hauger O, Sato Y, Araki H, et al: MR evaluation of the glomerular homing of magnetically labeled mesenchymal stem cells in a rat model of nephropathy. Radiology 238: 200-210, 2006.

7. Bos C, Delmas Y, Desmoulière A, et al: In vivo MR imaging of intravascularly injected magnetically labeled mesenchymal stem cells in rat kidney and liver. Radiology 233: 781-789, 2004.

8. Hoehn M, Küstermann E, Blunk J, et al: Monitoring of implanted stem cell migration in vivo: a highly resolved in vivo magnetic resonance imaging investigation of experimental stroke in rat. Proc Natl Acad Sci USA 99: 16267-16272, 2002.

9. Stutchfield BM, Forbes SJ and Wigmore SJ: Prospects for stem cell transplantation in the treatment of hepatic disease. Liver Transpl 16: 827-836, 2010.

10. Shabbir A, Zisa D, Suzuki G, et al: Heart failure therapy mediated by the trophic activities of bone marrow mesenchymal stem cells: a noninvasive therapeutic regimen. Am J Physiol Heart Circ Physiol 296: H1888-H1897, 2009.

11. Kraitchman DL, Heldman AW, Atalar E, et al: In vivo magnetic resonance imaging of mesenchymal stem cells in myocardial infarction. Circulation 107: 2290-2293, 2003.

12. Lee KD, Kuo TK, Whang-Peng J, et al: In vitro hepatic differentiation of human mesenchymal stem cells. Hepatology 40: 1275-1284, 2004.

13. Petersen BE, Bowen WC, Patrene KD, et al: Bone marrow as a potential source of hepatic oval cells. Science 284: 1168-1170, 1999.

14. Modo M, Cash D, Mellodew K, et al: Tracking transplanted stem cell migration using bifunctional, contrast agent-enhanced, magnetic resonance imaging. Neuroimage 17: 803-811, 2002.

15. Hung SC, Deng WP, Yang WK, et al: Mesenchymal stem cell targeting of microscopic tumors and tumor stroma development monitored by noninvasive in vivo positron emission tomography imaging. Clin Cancer Res 11: 7749-7756, 2005.

16. Shichinohe H, Kuroda S, Lee JB, et al: In vivo tracking of bone marrow stromal cells transplanted into mice cerebral infarct by fluorescence optical imaging. Brain Res Brain Res Protoc 13: 166-175, 2004.

17. Weissleder R: Molecular imaging: exploring the next frontier. Radiology 212: 609-614, 1999.

18. Daldrup-Link HE, Rudelius M, Oostendorp RA, et al: Targeting of hematopoietic progenitor cells with MR contrast agents. Radiology 228: 760-767, 2003.

19. Togel F, Hu Z, Weiss K, et al: Administered mesenchymal stem cells protect against ischemic acute renal failure through differentiation-independent mechanisms. Am J Physiol Renal Physiol 289: F31-F42, 2005.

20. Yocum GT, Wilson LB, Ashari P, Jordan EK, Frank JA and Arbab AS: Effect of human stem cells labeled with ferumoxides-poly-L-lysine on hematologic and biochemical measurements in rats. Radiology 235: 547-552, 2005.

21. Crabbe A, Vandeputte C, Dresselaers T, et al: Effects of MRI contrast agents on the stem cell phenotype. Cell Transplant 19: 919-936, 2010.

22. Suh JS, Lee JY, Choi YS, et al: Efficient labeling of mesenchymal stem cells using cell permeable magnetic nanoparticles. Biochem Biophys Res Commun 379: 669-675, 2009.

23. Chen YC, Hsiao JK, Liu HM, et al: The inhibitory effect of superparamagnetic iron oxide nanoparticle (Ferucarbotran) on osteogenic differentiation and its signaling mechanism in human mesenchymal stem cells. Toxicol Appl Pharmacol 245: 272-279, 2010. 